\title{
Cambios anatómicos en la corteza de Parkinsonia PRAECOX (RUIZ ET PAVÓN) HAWKINS CAUSADOS POR LA EPÍFITA TILLANDSIA RECURVATA L. (BROMELIACEAE)
}

\author{
luis Enrique Páez-Gerardo', Silvia Aguilar-Rodríguez², Teresa Terrazas³, Ma. Elena \\ HUIDOBRO-SALAS ${ }^{1}$ Y ERNESTO AGUIRRE-LEÓN ${ }^{1}$
}

\author{
'UBIPRO, FES-Iztacala, Universidad Nacional Autónoma de México, Av. de los Barrios 1, Los Reyes Iztacala, \\ Tlalnepantla 54090, A.P. 314, Edo. de México, México. \\ Correo-e: eall@servidor.unam.mx \\ ${ }^{2}$ Unidad de Morfología y Función, Laboratorio de Botánica, FES-Iztacala, Universidad Nacional Autónoma de \\ México, Av. de los Barrios 1, Los Reyes Iztacala, Tlalnepantla 54090, A.P. 314, Edo. de México, México. \\ ${ }^{3}$ Programa de Botánica, Colegio de Postgraduados, Montecillo, Edo. de México, 56230, México.
}

\begin{abstract}
Resumen: Tillandsia recurvata L. crece comúnmente sobre las ramas y tronco de Parkinsonia praecox (Ruiz et Pavón) Hawkins en la zona semiárida del Valle de Zapotitlán Salinas, México, y esta relación parece afectar al forofito de diferentes maneras. El propósito de este estudio fue evaluar el efecto que tiene el establecimiento de T. recurvata sobre los tejidos de la corteza en el forofito $P$. praecox. El estudio anatómico de la corteza permitió reconocer la presencia de floema no colapsado, floema colapsado, un córtex cuyas células bajo la epidermis muestran cloroplastos y una epidermis múltiple cubierta por una capa de cera epicuticular gruesa. Las raíces de T. recurvata no penetran los tejidos; sin embargo, en las zonas donde la epífita se presenta, se desarrolla un súber de reacción bajo la epidermis. El súber de reacción tiene por lo general un papel de protección contra infecciones, pero posiblemente también afecta negativamente la movilización de dióxido de carbono hacia los tejidos internos o la disipación de calor y luz. Estos aspectos parecen ser más importantes que la disminución de la fotosíntesis en las ramas donde la epífita se establece. Es probable que el sobrepeso que ocasiona el elevado número de individuos de Tillandsia recurvata sobre las ramas impida el desarrollo arquitectónico óptimo del forofito Parkinsonia praecox.
\end{abstract}

Palabras clave: anatomía, corteza, forofito, Parkinsonia praecox, Tillandsia recurvata.

\begin{abstract}
Tillandsia recurvata L. is commonly found growing on the branches and trunk of Parkinsonia praecox (Ruiz et Pavón) Hawkins in the semi-arid area of the Zapotitlán Salinas Valley, Mexico, and this relationship seems to affect the phorophyte in different ways. The purpose of this study was to evaluate the effect of the establishment of $T$. recurvata on bark tissues of the phorophyte P. praecox. The anatomical study of the bark allowed to recognize the presence of non-collapsed phloem, collapsed phloem, a cortex whose cells under the epidermis show chloroplasts and a multiple epidermis covered by a wide epicuticular wax layer. Tillandsia recurvata roots never penetrate the tissues. However, in those zones where the epiphyte occurs a wound periderm develops below the epidermis. Wound periderm provides protection against infection, but it probably also affects negatively the mobilization of carbon dioxide toward internal tissues or the dissipation of heat and light. These aspects seem to be more important than the photosynthesis decrease in branches where the epiphyte establishes. It is likely that the overweight caused by the large number of Tillandsia recurvata individuals on the branches prevents the adequate architectural development of the phorophyte Parkinsonia praecox.
\end{abstract}

Key words: anatomy, bark, phorophyte, Parkinsonia praecox, Tillandsia recurvata.

$\mathbf{L}$ as ramas de los árboles pueden soportar diversos tipos de plantas epífitas y es común considerar que éstas sólo utilizan al árbol como forofito, pues no penetran sus tejidos (Benzing, 1980). La epífita Tillandsia recurvata L., conocida como musgo bola o "paixtle", crece sobre numerosos forofitos (Hietz y Hietz-Seifert, 1994) (figura 1a) y el árbol Parkinsonia praecox (Ruiz et Pavón) Hawkins es su forofito común en la zona semiárida intertropical del Valle de Zapotitlán Salinas, en México. Esta relación parece producir diferentes efectos negativos sobre el hospedero, pues 
se sabe que T. recurvata causa al menos un parasitismo de tipo estructural, evidenciado por la afectación en la producción de brotes nuevos, en la arquitectura, el crecimiento y la reproducción del forofito (Montaña et al., 1997; PáezGerardo, 2005). Sin embargo, no se conocen investigaciones que revelen los posibles cambios anatómicos de la corteza de $P$. praecox derivados de la interacción con $T$. recurvata; de hecho, este tipo de estudios han sido pobremente abordados y sólo se han estudiado algunos aspectos morfológicos de la corteza relacionados con el establecimiento de semillas de Tillandsia (Samaniego, 2002). Por ello, nuestro objetivo fue evaluar a través de un análisis histológico el efecto del establecimiento de Tillandsia recurvata sobre la corteza del forofito Parkinsonia praecox.

\section{Materiales y métodos}

Para realizar el estudio anatómico de la corteza del forofito, se estudiaron diez individuos de Parkinsonia praecox que crecen en el Valle de Zapotitlán Salinas, en la región fisiográfica Tehuacán-Cuicatlán, entre los estados de Puebla y Oaxaca ( $97^{\circ} 24^{\prime} 50^{\prime \prime}$ longitud O y $18^{\circ} 27^{\prime} 30^{\prime \prime}$ latitud $\mathrm{N}$; altitud 1,678 m). El clima de la región corresponde al tipo BSo hw" (i'), es decir, seco semicálido, con una temperatura media anual de $21^{\circ} \mathrm{C}$ y una precipitación media anual de 400 a $500 \mathrm{~mm}$. El tipo de vegetación dominante es el matorral xerófilo. Se obtuvieron muestras de ramas de 4$5 \mathrm{~cm}$ de diámetro de Parkinsonia praecox sin la presencia de la epífita, así como muestras de ramas que la alojaban. Se describió la morfología de la corteza de acuerdo con la terminología propuesta por Barajas-Morales y PérezJiménez (1990).

Las muestras se fijaron en formol-alcohol-ácido acético glacial (0.5:9:0.5) (Johansen, 1940; Aguilar-Rodríguez, 1998) y se transportaron al laboratorio; después de 48 horas fueron lavadas y finalmente depositadas en una solución de glicerina-alcohol-agua (1:2:3) hasta que fueron seccionadas. Se realizaron cortes histológicos de $30 \mu \mathrm{m}$ de grosor en las secciones transversal y radial con un micrótomo de deslizamiento. Algunas secciones fueron aclaradas con hidróxido de sodio al $5 \%$ hasta que se perdieron los colores obscuros y el tejido quedó blanquecino. Posteriormente, se lavaron y se blanquearon con cloro comercial diluido al 50\%; las secciones aclaradas y no aclaradas fueron teñidas con safranina-verde rápido y montados en resina sintética (Johansen, 1940). Con la finalidad de conservar la capa de cera que cubre la corteza, otras secciones sólo se lavaron en agua y fueron montados directamente en gelatina glicerinada adicionada con safranina, cristal violeta o azul de anilina (Curtis, 1986; AguilarRodríguez, 1998). Para observar los elementos de tubo criboso y otras estructuras celulares que componen la corteza, se obtuvieron disociados de la misma empleando la técnica sugerida por Peterson y Blais (1991). Se cuantificó la lon- gitud y el diámetro del elemento de tubo criboso y se contó el número de áreas cribosas por elemento; se realizaron 25 mediciones de cada carácter por muestra con el analizador de imágenes Leica Qwin (Leica Microsystems, 2002). La descripción anatómica de la corteza se realizó de acuerdo con Trockenbrodt (1990).

\section{Resultados}

Descripción morfológica. La corteza externa de Parkinsonia praecox es lisa a ligeramente rugosa, con pliegues visibles a simple vista, de color verde amarillento. Es delgada, con un grosor total de $1.5 \mathrm{~mm}$ (figuras $1 \mathrm{~b}$ y $1 \mathrm{c}$ ).

Descripción anatómica. La anatomía de la corteza carente de la epífita presenta una zonificación de tejidos: floema no colapsado, floema colapsado, córtex y una epidermis múltiple (figura 1d).

El floema no colapsado varía de 80 a $142 \mu \mathrm{m}$ de grosor, mientras que el floema colapsado mide de 471 a $707 \mu \mathrm{m}$; ambos se componen de elementos de tubo criboso (ETC), células acompañantes (CA), parénquima axial (PA) y radios $(\mathrm{R})$ con abundantes cristales prismáticos. Los elementos de tubo criboso en esta especie son de pared delgada, de forma cuadrangular a irregular vistos en sección transversal y están organizados al azar, con un diámetro tangencial promedio de $20 \pm 0.26 \mu \mathrm{m}(18-26 \mu \mathrm{m})$ y una longitud promedio de $288 \pm 48 \mu \mathrm{m}(160-390 \mu \mathrm{m})$. La placa cribosa es compuesta, oblicua y con 3 a 13 áreas cribosas. Las células acompañantes tienen paredes delgadas y están generalmente distribuidas en las esquinas o las paredes radiales de los ETC. El parénquima axial se organiza entre los ETC y las CA, formando hileras tangenciales uniseriadas y cortas de 4 a 7 células; en la zona de floema colapsado estas hileras son muy evidentes dado su mayor tamaño cerca del córtex (figura 1e). En esta zona las agrupaciones de parénquima axial comúnmente contienen cristales prismáticos y tienen hasta tres estratos de células de mayor diámetro que se fusionan con el parénquima radial. Se observan dos tipos de radios en la sección transversal: los que tienen el mismo ancho en todo su curso y los que se dilatan marcadamente conforme se alejan de la zona cambial (figura 1f). Los primeros siguen un curso rectilíneo en el floema no colapsado y sinuoso en el floema colapsado; en esta región los segundos se abren en forma de abanico y en vista transversal ocupan una longitud de 48 a 86 $\mu \mathrm{m}$, con 71 a $94 \mu \mathrm{m}$ de ancho.

El córtex presenta bandas alternas de esclerénquima y parénquima. En las bandas de esclerénquima hay células de forma isodiamétrica fuertemente lignificadas, fibras y células de parénquima con paredes delgadas y lumen ocluido por cristales prismáticos; en las bandas de parénquima, las células tienen paredes primarias, observándose que las más cercanas a la epidermis presentan cloroplastos (figura 2c) y 
las más internas granos de almidón; además, con el aumento en el diámetro del córtex se expanden y se dividen. La epidermis presenta las células más externas en forma de papilas (figura 2b). Esta epidermis es múltiple con abundantes divisiones celulares periclinales y oblicuas, lo que le confiere un relieve irregular cubierto por una capa de cera muy gruesa (> $50 \mu \mathrm{m}$ ) (figura $2 \mathrm{a}$ ).

En la corteza del forofito Parkinsonia praecox, donde se establece Tillandsia recurvata, las raíces no penetran los tejidos (figura 2d). En posición subepidérmica y a partir del parénquima cortical se desarrolla un súber de 300 a $400 \mu \mathrm{m}$ de espesor, formado por dos capas, cada una con 3 a 4 hileras de células muertas, dilatadas en sentido radial y con inclusiones cristalinas, paredes finas y lumen amplio (figura 2e). Estas capas alternan con una banda angosta de dos hileras de células radialmente comprimidas con paredes gruesas que contienen sustancias oscuras (figuras $2 \mathrm{~d}$ y $2 \mathrm{e}$ ). Por encima del súber hay un cúmulo de células con contenidos oscuros (figura 2d). A diferencia de la corteza carente de la epífita, aquí no se observan las células epidérmicas en forma de papila ni la capa de cera que las cubren.

\section{Discusión}

Parkinsonia praecox ha sido reconocido como el forofito principal de Tillandsia recurvata en el área de estudio (Montaña et al., 1997; Luna-Cozar y Jones, 2002; PáezGerardo, 2005). Estas plantas se fijan principalmente en ramas rugosas de 2 a $8 \mathrm{~cm}$ de diámetro y parece ser que los pliegues que existen en la corteza rugosa y las ceras son atributos que facilitan la fijación de las semillas de T. recurvata. Sin embargo, Samaniego (2002) menciona que no existen diferencias en su establecimiento, pues éstas se pueden alojar tanto en ramas lisas como rugosas de Prosopis laevigata, árbol que también comparte el hábitat con Parkinsonia praecox. Es difícil explicar adecuadamente la fijación de la semilla, ya que en $P$. praecox se ha observado que factores como el viento, el agua y la gravedad, así como las ceras de la corteza, arrastran a las semillas a la posición lateral e inferior de las ramas, favoreciendo una mayor captación de agua y protección contra la incidencia solar (Luna-Cozar y Jones, 2002; García-Suárez et al., 2003). Probablemente también los pelos articulados de la semilla contribuyan a su fijación (Páez-Gerardo, 2005).

Con respecto al estudio anatómico de Parkinsonia praecox se observó que en las superficies carentes de Tillandsia, la corteza se compone en la parte más superficial de una epidermis múltiple, como lo menciona Roth (1981). De acuerdo con esta autora, las cortezas de algunas especies de árboles de zonas desérticas, como Parkinsonia, no forman una peridermis, por lo que la epidermis, igual que el córtex, se conservan en los individuos aunque exista un crecimiento secundario. Actualmente, dos de los autores (S. Aguilar y T. Terrazas) están desarrollando un estudio sobre la ontogenia de este tejido. Esta epidermis no se presenta de manera uniforme sobre la superficie de la rama y está formada por células dispuestas en forma de tiras. De acuerdo con Nilsen (1995), esta disposición favorece la penetración de la luz hacia los cloroplastos en el córtex. Al respecto, Roth (1981) menciona que existe tejido fotosintético y una hipodermis que se localiza en la capa más externa de este tejido verde. En este trabajo fue más clara la presencia de parénquima con cloroplastos por debajo de la epidermis múltiple. Con respecto a la función fotosintética de estos tallos verdes, en numerosas investigaciones se ha medido su magnitud (Nilsen, 1995) y al parecer el porcentaje neto de fotosíntesis es nulo, e incluso ligeramente negativo, por lo que el papel de la fotosíntesis cortical parece estar más relacionado con la reutilización del dióxido de carbono que libera a través de la respiración el tejido no fotosintético (Nilsen, 1995). Cozzo (1951) ha señalado que el tejido fotosintético degenera en ramas gruesas. Un estudio fisiológico preliminar de la corteza de Parkinsonia ha revelando que la tasa fotosintética, medida con un analizador de gases infrarrojo (IRGA), es nula; asimismo, la cuantificación de clorofilas indica la presencia de pigmentos accesorios cuya función principal es la disipación de calor y luz y no la fotosintética (C. Flores y J. Vázquez, com. pers.).

En las áreas de las ramas donde se desarrolla T. recurvata se observaron algunas modificaciones anatómicas. A pesar de que las raíces de la epífita no penetran los tejidos del forofito, la epidermis se ve afectada. Inmediatamente por debajo de ella, en el parénquima más externo, se llevan a cabo divisiones mitóticas, induciendo la formación de un súber de lesión que separa la epidermis de los tejidos internos, por lo que en estas zonas se pierden el tejido epidérmico y el fotosintético. Roth (1981) menciona que cualquier planta puede formar una peridermis como una respuesta a daños. En esta zona también se observa una banda de células llenas de sustancias oscuras, posiblemente compuestos fenólicos, que Fahn (1974) define como resinas o taninos y que se forman como una respuesta contra el ataque de parásitos o cualquier otro organismo dañino. Páez-Gerardo (2005) encontró que después de una lluvia intensa el peso de carga del forofito puede aumentar hasta $68 \%$, por lo que la concentración de humedad propiciaría el desarrollo más extensivo de súber de reacción con taninos que impiden la entrada de patógenos (Bryant y Raffa, 1995).

Finalmente, se puede decir que la alta densidad poblacional de la epífita, correspondiente al número de conjuntos coloniales o "bolas" por árbol, se incrementa hasta diez veces en $P$. praecox de áreas deterioradas (Páez-Gerardo, 2005). Este incremento constituye un parasitismo estructural, pues el hecho de tener más plantas de la epífita, trae consigo la formación de súber de reacción en amplias superficies de las ramas. Probablemente, la presencia de súber disminuya la funcionalidad del mecanismo de movi- 
lización de dióxido de carbono hacia tejidos internos o la disipación de calor y luz (C. Flores y J. Vázquez, com. pers.), afectando negativamente la carga de energía óptima de estos individuos en ambientes con alta incidencia de luz (Nilsen, 1995).

Montaña et al. (1997) mencionan que el parasitismo estructural causa una disminución importante en la fotosíntesis en las ramas donde se implantan las epífitas. En nuestra opinión, parece ser que la alta incidencia de epífitas es importante en la formación de cambios anatómicos de la corteza, lo que disminuye la movilización del $\mathrm{CO}_{2}$, afectando más los procesos respiratorios que los fotosintéticos. La alta densidad de Tillandsia recurvata en las ramas y el sobrepeso son factores adicionales que afectan el desarrollo estructural óptimo del forofito Parkinsonia praecox.

\section{Agradecimientos}

Los autores agradecen a Ma. Águeda Castro y Carmen de la Paz Pérez Olvera la revisión crítica del manuscrito y sus sugerencias, a Jorge Meave su interés y revisión minuciosa, a César M. Flores y Josefina Vázquez por haber discutido algunos aspectos de la fisiología de Parkinsonia praecox con base en algunos de sus datos no publicados, y a Gustavo I. Labrada por el apoyo técnico brindado. El presente estudio fue financiado a través del Programa de Apoyo a los Profesores de Carrera para la formación de Grupos de Investigación (PAPCA) 2003 de la FES-Iztacala, UNAM.

\section{Literatura citada}

Aguilar-Rodríguez S. 1998. Técnicas de laboratorio para el estudio de las embriofitas. En: Tejero-Díez J.D. y Granillo V.M.P. Eds. Plantae: Introducción al Estudio de las Plantas con Embrión, pp. 275-285, Universidad Nacional Autónoma de México, Campus Iztacala, Tlalnepantla. Edo. de México.

Barajas-Morales J. y Pérez-Jiménez L. 1990. Manual de Identificación de Árboles de Selva Mediante Cortezas. Instituto de Biología, Universidad Nacional Autónoma de México, México, D.F.

Benzing D.H. 1980. The Biology of the Bromeliads. Madison River Press, Eureka, Cal
Bryant P.J. y Raffa F.K. 1995. Chemical antiherbivore defense. En: Gartner L. Ed. Plant Stems: Physiology and Functional Morphology, pp. 365-381, Academic Press, San Diego.

Cozzo D. 1951. Anatomía del leño secundario de las leguminosas mimosóideas y cesalpinióideas argentinas. Revista del Instituto Nacional de Investigaciones. Ciencia Natural, Ciencia Botánica 2:63-146.

Curtis D.J. 1986. Microtecnia Vegetal. Trillas, México, D.F.

Fahn A. 1974. Anatomía Vegetal. H. Blume, Madrid.

García-Suárez M.D., Rico-Gray V. y Serrano H. 2003. Distribution and abundance of Tillandsia sp. (Bromeliaceae) in the Zapotitlán Valley, Puebla, México. Plant Ecology 166:207215.

Hietz P. y Hietz-Seifert U. 1994. Epífitas de Veracruz: Guía Ilustrada para las Regiones de Xalapa y Los Tuxtlas, Veracruz. Instituto de Ecología, A.C., Xalapa.

Johansen D.H. 1940. Plant Microtechnique. McGraw-Hill, Nueva York.

Leica Microsystems. 2002. Leica Qwin. Digital imaging systems for professional microscopy Leica. Imagic Bildverarbeitung AG, Wetzlar, Alemania.

Luna-Cozar J. y Jones W.R. 2002. Evaluation of ball moss, Tillandsia recurvata $\mathrm{L}$. as a refuge site for insects during the dry season in dry tropical forest.

<http://esa.confex.com/esa/2001/techprogram/paper_3969.htm> (consultado nov. de 2004)

Montaña C., Dirzo R. y Flores A. 1997. Structural parasitism of an epiphytic bromeliad upon Cercidium praecox in an intertropical semiarid ecosystem. Biotropica 29:517-521.

Nilsen T.E. 1995. Stem photosynthesis: extent, patterns, and role in plant carbon economy. En: Gartner L. Ed. Plant Stems: Physiology and Functional Morphology, pp. 223-240, Academic Press, San Diego.

Páez-Gerardo L.E. 2005. Biología de Tillandsia recurvata L. (Bromeliaceae) y su importancia en aplicaciones prácticas y ecológicas. Tesis de Licenciatura, Facultad de Estudios Superiores Iztacala, Universidad Nacional Autónoma de México, Tlalnepantla, Edo. de México, 82 pp.

Peterson C.A. y Blais A.M. 1991. A rapid method for macerating phloem. Biotechnique and Histochemisty 66:242-245.

Roth I. 1981. Structural patterns of tropical barks. Encyclopaedia of Plant Anatomy. Gebruder Borntraeger, Berlín.

Samaniego H.A. 2002. Tillandsia recurvata: relación con la textura de las ramas de Prosopis laevigata. Biotropica 24:402407.

Trockenbrodt M. 1990. Survey and discussion of the terminology used in bark anatomy. International Association of Wood Anatomists Bulletin new series 11:141-166. 

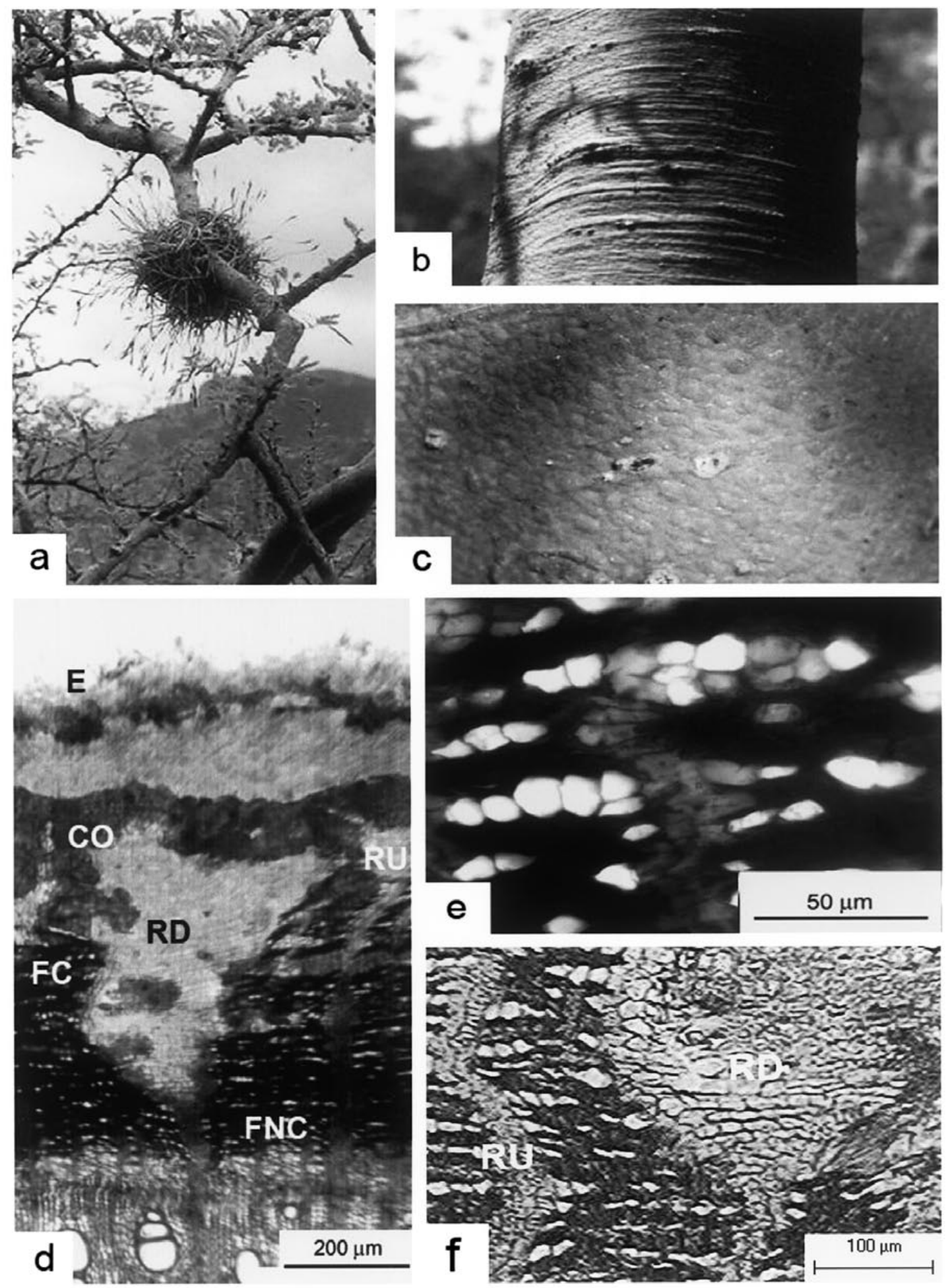

Figura 1. Parkinsonia praecox. a. Rama con una "bola" de Tillandsia recurvata. b. Corteza externa en fuste. c. Detalle de la corteza externa en fuste. d. Vista general de secciones transversales de la corteza en ramas de Parkinsonia praecox. e. Bandas tangenciales de parénquima axial. f. Radios dilatados y no dilatados. $\mathrm{E}=$ epidermis, $\mathrm{CO}=$ córtex, $\mathrm{FC}=$ floema colapsado, FNC = floema no colapsado, $\mathrm{RU}=$ radios no dilatados, $\mathrm{RD}=$ radios dilatados. 

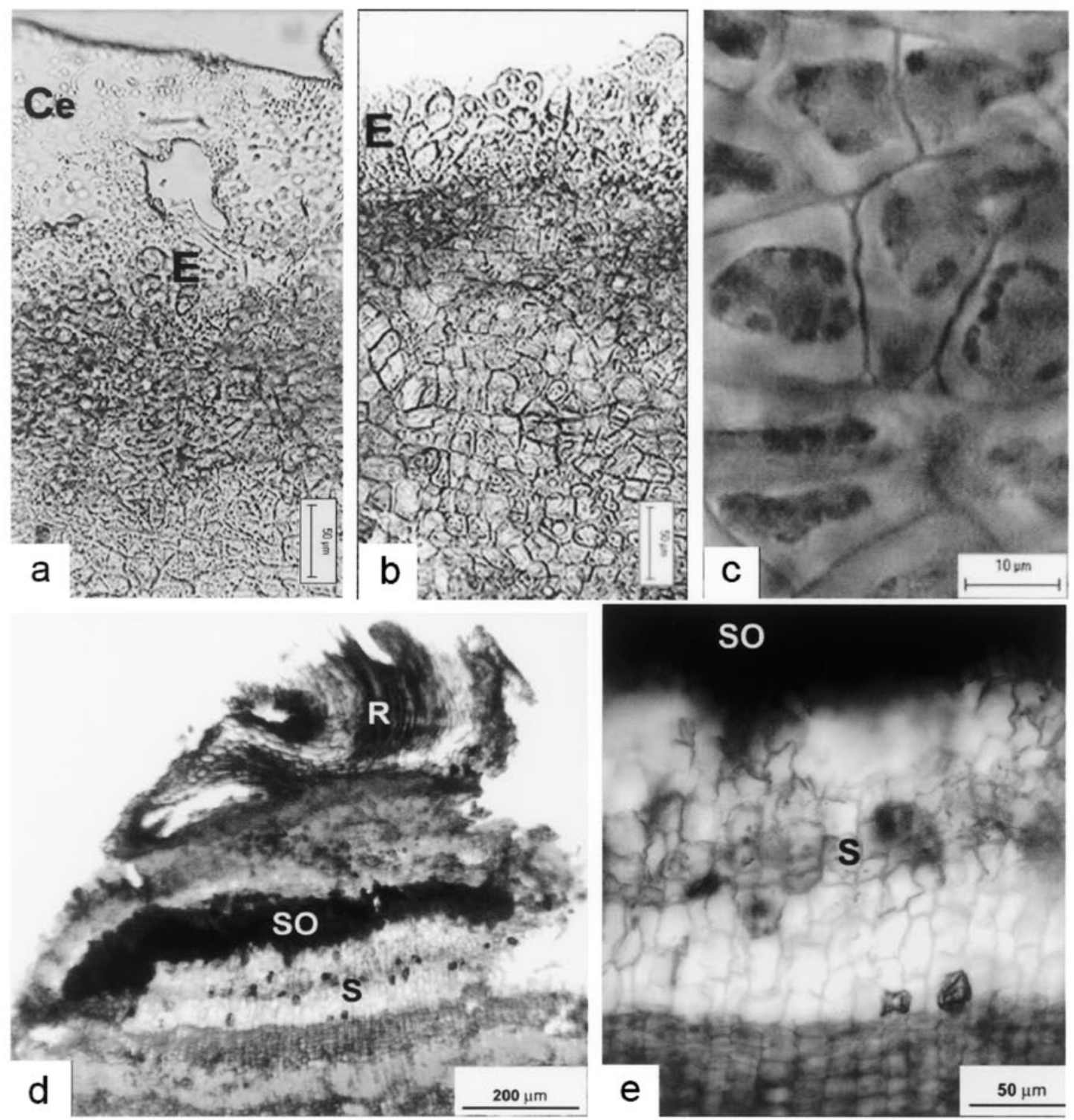

Figura 2. a. Sección transversal de la epidermis y el córtex en ramas de Parkinsonia praecox. Capa de cera que cubre las células epidérmicas. b. Epidermis múltiple. c. Células de parénquima con cloroplastos. d. Sección transversal de la zona de interacción de la raíz de Tillandsia recurvata con la corteza de rama de Parkinsonia praecox. Porción de la raíz en contacto con los tejidos modificados (súber). e. Células del súber dilatadas radialmente. $\mathrm{E}=$ epidermis, $\mathrm{Ce}=$ ceras. $\mathrm{R}=$ raíz de $T$. recurvata, $\mathrm{SO}=$ sustancias oscuras, $\mathrm{S}=$ súber de reacción. 